\title{
Framing the structural role of mathematics in physics lectures: A case study on electromagnetism
}

\author{
Ricardo Karam \\ Faculty of Education, University of Hamburg, 20146 Hamburg, Germany
}

(Received 27 September 2013; published 28 May 2014)

\begin{abstract}
Physics education research has shown that students tend to struggle when trying to use mathematics in a meaningful way in physics (e.g., mathematizing a physical situation or making sense of equations). Concerning the possible reasons for these difficulties, little attention has been paid to the way mathematics is treated in physics instruction. Starting from an overall distinction between a technical approach, which involves an instrumental (tool-like) use of mathematics, and a structural one, focused on reasoning about the physical world mathematically, the goal of this study is to characterize the development of the latter in didactic contexts. For this purpose, a case study was conducted on the electromagnetism course given by a distinguished physics professor. The analysis of selected teaching episodes with the software Videograph led to the identification of a set of categories that describe different strategies used by the professor to emphasize the structural role of mathematics in his lectures. As a consequence of this research, an analytic tool to enable future comparative studies between didactic approaches regarding the way mathematics is treated in physics teaching is provided.
\end{abstract}

DOI: 10.1103/PhysRevSTPER.10.010119

PACS numbers: 01.40.Fk, 01.40.gb

\section{INTRODUCTION}

Many physics instructors complain that their students do not know enough mathematics. However, it is quite clear that the domain of basic mathematics skills does not guarantee success in physics, since "using mathematics in physics is much more complex than the straightforward application of rules and calculation" [1]. Despite the deep interrelations between physics and mathematicsconfirmed by both historical and epistemological studies [2-7] - it is not uncommon in physics education to regard mathematics as a mere tool to quantify physical entities and express relations between them. This instrumental view of the role of mathematics leads to an artificial separation between the mathematical and the conceptual aspects of physical theories, which becomes evident when educators sustain that physics instruction should concentrate on the latter to the detriment of the former.

Previous research into the role of mathematics in physics education has focused mainly on the learning perspective by analyzing students' reasoning in thinking-out-loud problem-solving sections and interviews [8-12]. Some research outcomes highlight difficulties faced by students in transferring mathematical knowledge to physical contexts [13], assigning meaning to physics equations [14], using mathematical structures to model physical situations [15], and other tasks associated with a deep understanding

Published by the American Physical Society under the terms of the Creative Commons Attribution 3.0 License. Further distribution of this work must maintain attribution to the author(s) and the published article's title, journal citation, and DOI. of the interplay between physics and mathematics. But how are these difficulties related to instruction? If one observes, for instance, that students often focus on the instrumental role of mathematics when they solve problems (e.g., plug and chug), it is likely that this attitude is related to certain features of the instruction they had. Therefore, focusing on the role of mathematics in physics from the teaching perspective - a facet rather overlooked in current physics education research - should enhance our understanding of the origins of students' difficulties and provide possible solutions to overcome them. This assumption determines the guidelines of this work.

Taking the multifaceted character of the role(s) of mathematics in physics into account, an overall distinction between the technical dimension-associated with an instrumental (tool-like) use of mathematics-and the structural dimension-related to the use of mathematics as a reasoning instrument to think about the physical world (Sec. II) is proposed. The central aim of this research is to characterize the development of the structural dimension in physics teaching approaches. For this purpose, a case study was conducted on the electromagnetism course given by a distinguished professor in the undergraduate introductory level (Sec. III). The analysis of these lectures led to the identification of a set of categories to describe the professor's didactic discourse. These categories are described, justified, and exemplified in Sec. IV and then applied in the coding of two teaching episodes extracted from the lectures (Sec. V).

The intended contributions of this work are twofold. First, I characterize the didactic choices made by the professor to teach his students how to frame the physical world mathematically and illustrate the complexity of this 
process with several examples extracted from his electromagnetism lectures. Second, an analytic tool to enable future comparative studies between lectures (or lessons) regarding the way mathematics is treated in physics teaching is provided.

\section{TECHNICAL AND STRUCTURAL DIMENSIONS}

The intermediate portion of mathematical science, which consists of calculation and transformation of symbolic expressions, is most essential to physical science, but it is in reality pure mathematics. Everything connected with the original question may be dismissed from the mind during these operations, and the mathematician to whom they are referred may be doubtful whether his results are to be applied to solid geometry, to hydrostatics or to electricity. But as we are engaged in the study of Natural Philosophy we shall endeavour to put our calculations into such a form that every step may be capable of some physical interpretation, and thus we shall exercise powers far more useful than those of mere calculation-the application of principles and the interpretation of results (Ref. [16], p. 672, our emphasis).

This quotation-extracted from Maxwell's inaugural lecture given in 1860 at King's College-implies that, although mathematical calculations play an essential role in physics (natural philosophy at that time), physicists should constantly pursue physical interpretations of each step when performing them. This message illustrates an overall distinction between two ways of using mathematics in physics, namely, a technical and a structural one. Following Maxwell's quotation, the technical dimension is associated with the mathematical calculations without any connection with physical phenomena, whereas the structural one is related to the use of mathematics to reason about the physical world.

When translating this dichotomy (it is more likely a duality) into physics teaching and learning practices, it is possible to distinguish between the development of technical and structural skills [17-19], which implies different kinds of questions to be asked to students, different teaching approaches, assessments, materials, and so forth. In fact, the domain of technical skills has already been proven to be insufficient (although probably necessary) for success in physics courses [20]. The excessive focus on this instrumental dimension in instruction has had a major impact on students' lack of interest in physics [21] and has motivated physics education researchers to advocate in favor of a focus on conceptual physics. Nevertheless, the intrinsic mathematical nature of the physical sciences [4] reveals that understanding in physics is strongly connected with the ability to think about the world with mathematical structures (structural dimension). In this sense, Hestenes ([22], p. 104) stresses that "the challenge is to seriously consider the design and use of mathematics as an important subject for Physics Education Research.” Sherin ([8], p. 482) argues in the same direction by stating that "we do students a disservice by treating conceptual understanding as separate from the use of mathematical notations."

Physics education research literature provides various means to categorize students' different ways of using mathematics to solve physics problems, which seem to be broadly classifiable according to this general technical or structural distinction. Among them, Sherin's symbolic forms [8] certainly belong to the structural dimension, since they are connected with a deep understanding of how to represent conceptual schemata-which are very basic mathematical relations between physical quantities-in terms of equations. The epistemic games proposed by Tuminaro and Redish [9] could possibly be classified into the ones focusing merely on technical aspects (plug and chug and transliteration to mathematics) and the ones demanding a deeper or structural understanding (mapping meaning to mathematics and mapping mathematics to meaning). Similarly, the classification of Walsh et al. [10] of students' problem-solving approaches into a scientific (structural) and other less structured approaches like plug and chug and memory based (both technical) seem to fit in such an overall distinction. The categorization of Bing and Redish [11] of students' epistemological framing could also be separated into technical (calculating and invoking authority) and structural (physical mapping and mathematical consistency) approaches.

It is not the intention, of course, to imply that all the subtleties contained in each of these categories can be incorporated by such a broad distinction, but rather to focus on differentiating a superficial from a deep understanding of physics regarding the use of mathematics. After consulting several physics graduate students and faculty members, Chasteen et al. [23] identified this focus on such deep understanding (mathematical sophistication [23], p. 924) as one of the major learning goals of physics courses and an essential trait of what it means to "think like a physicist." Furthermore, the nontriviality of the ability to use mathematical structures to think about the physical world is explained by Redish [24] with the argument that math in physics is "semantically different" from simply doing math [25]. According to him, physicists make different use of constants and variables, put great value on dimensional analysis, and often blend conceptual physics with mathematical symbols when interpreting equations. (See also Ref. [31] for a comprehensive list of differences between math in physics and math in math.). With the purpose of presenting a synthetic view of this technical-structural distinction, Table I displays different epistemological ways to use mathematics in physics, as well as different views on this interplay, which are associated with each dimension. 
TABLE I. Technical-structural distinction concerning the role of mathematics in physics.

\begin{tabular}{lc}
\hline \hline Technical (instrumental, procedural) & Structural (relational, organizational) \\
\hline $\begin{array}{l}\text { Blindly use an equation to solve quantitative problems (plug and } \\
\text { chug) }\end{array}$ & $\begin{array}{c}\text { Derive an equation from physical principles using logical } \\
\text { reasoning }\end{array}$ \\
$\begin{array}{l}\text { Focus on mechanic or algorithmic manipulations } \\
\text { Focus on physical interpretations or consequences } \\
\text { Use arguments of authority; rote memorization of equations and } \\
\text { rules }\end{array}$ & $\begin{array}{c}\text { Justify the use of specific mathematical structures to model } \\
\text { physical phenomena }\end{array}$ \\
$\begin{array}{l}\text { Fragmented knowledge: memorize different equations for each } \\
\text { specific case (e.g., free fall and vertical throw) }\end{array}$ & $\begin{array}{c}\text { Structured knowledge: connect apparently different physical } \\
\text { assumptions through logic (e.g., Snell's law and Fermat's }\end{array}$ \\
$\begin{array}{l}\text { Identify superficial similarities between equations (e.g., } d=\frac{1}{2} g t^{2} \\
\text { and } K=\frac{1}{2} m v^{2} \text { both seen as "half a constant times a squared }\end{array}$ & $\begin{array}{c}\text { Recognize profound analogies and common mathematical } \\
\text { variable") }\end{array}$ \\
Mathematics seen as calculation tool & central force field) \\
Mathematics seen as "just another" language used to represent & Mathematics seen as reasoning instrument \\
and communicate & Mathematics seen as essential to define physical concepts \\
\hline \hline
\end{tabular}

As physics education researchers, we are all engaged in finding ways to enable our students to understand this difficult science deeply, which involves their ability to map meaning to mathematics and vice versa [9], blend conceptual and formal mathematical reasoning [12], adopt scientific approaches [10], think like physicists [23], or, in other words, recognize the structural role of mathematics in physical thought and apply sophisticated strategies consciously to problem solving. However, the truth is that we do not have clear guidelines on how to teach students to do that effectively. One reasonable possibility for finding such guidelines is to look for specific characteristics in the didactic discourses of excellent lecturers "in action." Based on this hypothesis, a case study was conducted on the electromagnetism lectures given by a distinguished professor of physics majors at the introductory level. Thus, the central research question of this study can be formulated as follows:

What are the main features of the didactic discourse of an experienced physics professor who focuses on the structural role of mathematics in his electromagnetism lectures?

In the search for an instrument to characterize the lectures, an analytic tool was developed, which is likely fruitful for the conduction of future comparative studies, although this hypothesis is not investigated here. The methodology used to analyze these lectures and the reasons for the selection of this particular case study are described in the next section.

\section{METHODOLOGY}

The methodological design of the research was outlined according to the goal of investigating the role of mathematical reasoning in physics lectures. Given the innovative character of this approach and the absence of categories for such analysis in the literature, conducting a case study [32] seemed the most appropriate alternative. According to Gerring ([33], p. 40) "case studies tend to be more useful when the subject being researched has not yet been explored in a systematic manner or when it is considered from a new perspective." Moreover, it is acknowledged that case studies can penetrate situations in ways that are not always susceptible to numerical analysis [34].

It is important to justify the decision of studying the lectures of this professor in particular. In fact, several reasons influenced this choice. The professor is quite well known in the Physics Department of the University of São Paulo for having a great ability to explain things clearly and for encouraging his students to reason about the physical meaning underneath the mathematical formalism. Furthermore, he has been teaching introductory courses for more than 30 years. Another motivation is related to the students' approval of his lectures. At the end of each semester, students were asked to rate the quality of the lectures according to the following criteria: (1) professor's interaction with the class, (2) preparation of the lectures, and (3) quality of explanations, using a 4-point Likert scale items (very good, $100 \%$; good, $75 \%$; regular, $50 \%$; poor, $25 \%$ ). It was possible to have access to students' evaluations of his courses since 2005, which makes a total of 317 students (the average number of students per class being 50). The approval of his lectures is attested by expressive numbers-99.13\% for (1), $99.53 \%$ for (2), and $98.82 \%$ for (3) - which makes him one of the best-evaluated professors in the department.

Thus, the starting research hypothesis was that the lectures of this particular professor would provide several examples of the emphasis on the structural dimension. If this were correct, it would be possible to better characterize the focus on this dimension in didactic situations. In order to confirm the validity of this hypothesis, 10 lectures of the professor on special relativity were recorded. This pilot 
study revealed the excellent quality of his explanations and left no further doubt concerning the appropriateness of a deep investigation of his lectures.

Moreover, both the analysis of the electromagnetism course and the interviews provide an a posteriori validation for this choice. In one the interviews, for instance, the professor clearly explains why he does not emphasize the technical dimension in his lectures. In his words, "You cannot learn the technique at once; practice is really important. I feel that it is not possible to learn this in the lecture. [...] Thus, what I prefer to do in my lectures is to explore the multidimensionality of the physical knowledge and exercise a bit each of its dimensions. In reality, my hope is that they become aware of this broad picture."

The research data of the main study consist of the recordings of 40 lectures (total of approximately 60 hours of video) from an introductory course [35] on electromagnetism given for physics majors in 2009 [36]. The camera was directed toward the professor and special attention was given to the moments when mathematical reasoning took part in the exposition. The textbook used in the course is a compendium of lecture notes on electromagnetism elaborated by the professor and other colleagues through the years. The 40 chapters of the textbook are related to the (ideal) program of the 40 lectures to be given in the course.

The compendium of lecture notes has quite a different style, compared with traditional introductory level physics textbooks (e.g., Refs. [37-40]). The main features that distinguish them are as follows: (1) intense presence of epistemological discussions (e.g., What is physics? What is the relationship between physics and mathematics? How does electromagnetic theory enable us to reason about the world?), as well as dictionary definitions of essential terms (e.g., law, principle, explanation), (2) few examples (typical problems) are presented and discussed in depth, each step being carefully justified in their resolution, (3) a small number of problems and conceptual questions are proposed at the end of each chapter. Students are encouraged to use this material as support for individual study, but it is not required for the attendance of the lectures.

The evaluation of the course was carried out by means of four individual exams throughout the semester. The final average score $\mathrm{M}$ is calculated as follows [41]: $M=0.3 \times$ (sum of the two highest grades among the first three exams) $+0.4 \times$ (grade on the fourth exam). Among the 81 students enrolled at the beginning of the period analyzed, 11 left it after the first exam, and 59 of the remaining 70 were approved. Even though a systematic comparative study was not conducted, $84 \%$ of approval can be considered a successful result considering the history of students' performance in the first course on electromagnetism.

A preliminary analysis of the course syllabus pointed out moments in which the intertwined relationship between mathematics and physics would be addressed. Central concepts like charge, density, flux, and electric current would be introduced and properly mathematized. Mathematical operations such as derivatives, multiple integrals, vector operators (gradient, divergence, and curl), among others, would be physically interpreted in the context of charges and fields. Each one of Maxwell's equations would be addressed in their integral and differential forms.

After watching all of the lectures, 19 teaching episodes were selected for further analysis. This selection followed mainly two criteria: (1) the relevance and degree of mathematization of the topic (including core concepts like charge density, electric flux, current, field; physical interpretations of mathematical operations, and the explanation of Maxwell's equations) and (2) moments when students made interventions that indicated difficulties in understanding the use of the mathematical formalism of electromagnetism. The 19 teaching episodes were divided into conceptual explanations and problem solving and their detailed analysis can be found in Ref. [42].

The software Videograph [43] was used for the analysis of the teaching episodes. This tool allows one to categorize time intervals of teaching and learning situations (in this case the lectures) and to generate timelines that dynamically illustrate how the explanations evolved during the course of an episode. An important step for this kind of structured observation is to define a time unit for the categorization [44]. After a few attempts, 20 seconds seemed sufficient for the desired level of precision. Figure 1 illustrates the software's interface during the coding process. It shows the three windows (video, timeclip, and codeview) with which one works simultaneously when coding. At the exact moment displayed in Fig. 1, the 20 seconds between 13:20 and 13:40 were being transcribed and categorized. Those 20 seconds were categorized as mathematizing (M1-assumptions), analogy (A1-material), and visual representations (V1—gestures). The categories of analysis are properly presented, exemplified, and explained in Sec. IV.

It is important to mention that working with Videograph was essential for the very process of finding the most suitable categorization system to analyze the lectures. The visual timelines plotted by the program were helpful in presenting and discussing the pertinence of the categories with other colleagues. Aiming at minimizing the degree of subjectivity in the categorization, two sessions of validation were conducted. The sessions were organized as follows: First, the categories were briefly described and the categorization of a small lecture excerpt was presented and justified. Then, five fellow researchers (four graduate students and one professor) categorized five lecture passages (each one about 7 minutes long) individually using the software Videograph. Finally, the categorizations made by them were compared with what I had previously coded. 


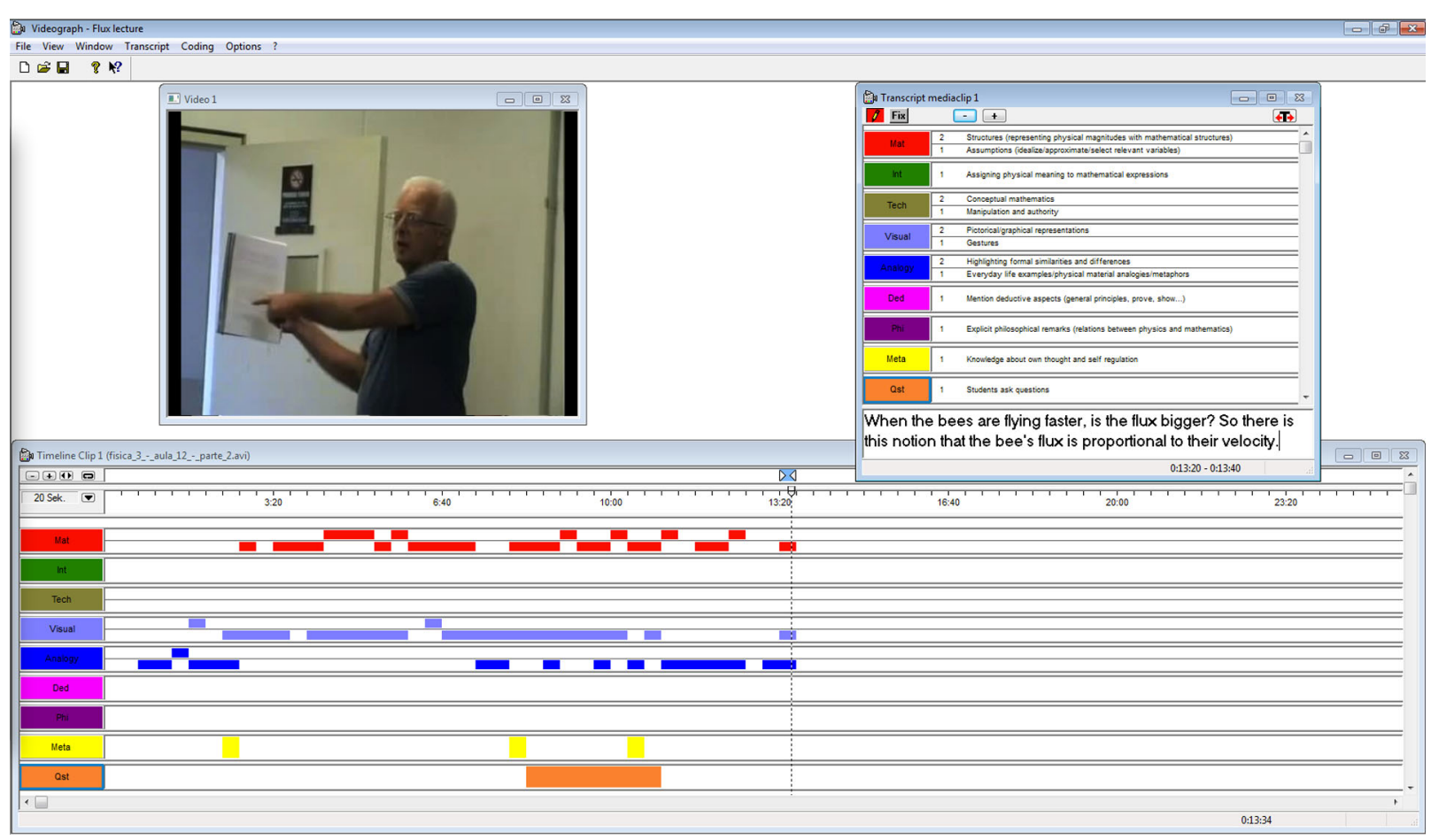

FIG. 1. Videograph's coding interface.

The initial comparison already revealed a satisfactory level of agreement $(60 \%-70 \%)$, which was increased (to 80\%) after a collective discussion on the interpretations and criteria utilized.

Naturally, one must be aware of the fact that the didactic discourse conducted in a classroom environment is intentional, dependent on the context and on the audience, and is not necessarily a reflection of the way a lecturer thinks. Therefore, in order to investigate the professor's intentions and conceptions, two semistructured interviews (totaling approximately 2 hours) were conducted with him. These interviews were important not only to provide a better understanding of the professor's intentions behind his actions, but also for the internal validation of the categorization system.

\section{CATEGORIES OF ANALYSIS}

The process of reaching the categorization system was mostly influenced by three domains: (1) historical and epistemological studies on the relationship between physics and mathematics [2-7], (2) categories found in the physics education literature (classification of students' reasoning while solving problems) [8-15,23], and (3) the dynamic process of watching the lectures and searching for appropriate categories while using the software Videograph.

For organizational reasons, the nine categories used for the analysis are divided into three subsets that encompass complementary dimensions of the lectures. The first three categories (mathematizing, interpreting, and technical) are directly related to the way mathematics is being treated. The next three categories (visual representations, analogies, and deduction) address a set of common pedagogical and linguistic strategies used by the professor to focus on translation processes (i.e., from physics to mathematics and vice versa). The last three (philosophy, metacognition, and questions) locate instants in which the "normal course" of a lecture is interrupted by explicit moments of meta-level discussions. In the following, a detailed description of the categories is presented and each of them is exemplified with excerpts from the teaching episodes.

\section{A. First dimension: Translation processes and technical use}

The three categories of the first dimension refer specifically to how mathematics is being used in the physics lectures. This dimension contains both translation processes (from physics to mathematics and vice versa) and moments of pure technical use of mathematics. Because they characterize considerably different approaches, these categories are thought to be mutually exclusive, even though at certain moments the time unit (20 s) is not small enough to be able to capture shifts.

\section{Mathematizing}

Mathematizing is understood as the process of constructing a mathematical representation for a physical situation (in the broad sense). This process can be seen as a translation from the physical world (e.g., observations 
and experiments) into mathematical structures (e.g., numbers, functions, and vectors). Being successful in this translation depends on the ability to see the world through mathematical lenses, which involves not only a significant understanding of mathematical concepts and theories, but also the ability to abstract, idealize, and model the physical reality. In fact, this is an extremely complex process and can be easily ignored in physics instruction. If a lecturer, for instance, starts his/her presentation of a concept by introducing its formula, it is likely that the whole mathematization (thought as a process) is already disregarded. In the lectures analyzed, the professor gave special attention and devoted considerable time to mathematization. This was conducted explicitly during the introduction of a new idea or concept and in the beginning of problem-solving activities.

Mathematizing a physical situation inevitably passes by the construction of idealized models that abstract some complexities of the real world. As Kline [45] points out, Galileo's approach to free fall was essentially mathematical, since he built an idealized representation of the situation by eliminating some effects (e.g., friction and air resistance) and focused on quantitative relations in order to obtain its basic principles [46]. Therefore, adopting this broader notion of mathematization, the first step of building the model was considered one of its subcategories (M1assumptions). In the lectures, this happened when the professor explicitly mentioned approximations, idealizations, and selection of relevant variables, as illustrated in the following excerpt [47]:

You can approximate one road as a one-dimensional thing. Its length is much bigger than its width. One strand of hair, you can also approximate it as a onedimensional thing. One strand of hair has only one dimension? No! It has 3. (lecture 5-36:40-37:00).

Furthermore, at this early stage of building a model, the professor usually draws attention to the distinction between reality and its representations. This attitude is evident in the following statement:

Does an electrical wire have charge density? [...] You take this wire here and cut it with your mind. This $d x$ is not a physical cut. You cut it with your mind, mathematically. (lecture 5-41:40-42:00).

The second subcategory of mathematizing is related to the actual process of finding a suitable mathematical structure to represent a physical situation or concept (M2-structures). Some philosophers use the metaphor prefabricated mathematics and compare the attitude of a physicist to "a person who goes to the market of mathematics to take what he/she needs to construct his/her theory" ([7], p. 83). Representing continuous quantities by real numbers, oriented quantities by vectors, and quantum states by matrices are some examples of the uses of prefabricated mathematics. The following passage exemplifies this search for suitable mathematical structures to model physical phenomena:

But the problem is that $i$ [electric current] is a scalar. And if I want to describe the situation more precisely I have to use a vector. [...] I will use a vector pointing in the velocity direction, so that if the charge is going in this direction and I put the surface here [gestures] it represents the notion that it does not penetrate the surface. And then we will use the well-known concept of flux. (lecture 26-22:40-23:40).

There is an important aspect that has to be emphasized here. Understanding the mathematization process in depth involves one being able to identify the essential aspects of the physical situation that justify the use of a particular mathematical structure. Similarly, Redish remarks that to construct a mathematical representation of a physical system, "we have to understand what mathematical structures are available and what aspects of them are relevant to the physical characteristics we are trying to model" ([24], p. 7). In this sense, it should not be enough just to know how to operate with the mathematical "tools" (functions, logarithms, matrices, vectors, etc.). It is important to identify the essential aspects of these structures to model physical phenomena. As exemplified in the quotations above, making this point explicit was a major focus in the lectures. Moreover, it represents an essential skill concerning the structural role of mathematics in physics (Sec. II).

\section{Interpreting}

The first category illustrated the process of going "from physics to mathematics" whereas the second concerns the opposite direction. After presenting or deriving a mathematical expression-during either the explanation of a concept or problem solving - the focus was immediately directed to the physical interpretation of its meaning. A formula was rarely presented or derived without a careful explanation of its physical meaning. This was done with the aid of powerful schemes and an intense use of visual representations (figures, diagrams, gestures). In general, the focus on the interpretation occurred after the solution of a problem, in which a mathematical expression was obtained. Sentences like "What does this expression mean?" or "What does this equation say?" denote this approach as exemplified on the following excerpts.

Why $Q^{2}$ appears in this expression? Does the charge $Q$ interact with itself? Look what I did here. I picked up a charge and brought it here [gesture]. And it was interacting with what was already there. [...] This is what self-energy means. There is a charged system, but 
this charge interacts with it and this is stored in that expression. (lecture 21-29:00-30:00).

Today we do not want to calculate the divergence, but to apply it in a physical situation. The divergence is a mathematical machine. What favor does it do for you? It finds the charge distribution, ok? If you calculate the divergence in a point with no charge density you get zero, right? On the other hand you expect it to be nonzero where it finds charge. (lecture 23-1:29:201:30:20).

In these passages the professor emphasizes the importance of looking for physical interpretations in mathematical expressions. In the first quotation the term $Q^{2}$-present in the expression of the self-energy of a charged sphere-is properly interpreted and justified with physical arguments. In the second, a physical interpretation for the divergence operator is given when the professor states its "mathematical favor" of finding points with nonzero charge density.

One very common strategy to interpret mathematical expressions physically is the analysis of special cases, as exemplified next.

What do you expect from the expression if you have a positive charge here, a negative charge here [gestures], and move along the y-axis? To which direction should the field point? [...] What happens if $x, y$ or $z$ go to 0 ? Does it make sense? [...] If you don't expect anything from an equation you are lost. (lecture 9-58:00$58: 40+1: 00: 00-1: 00: 20)$.

After obtaining a mathematical result to a problem, the professor often encouraged the students to check its consistency by analyzing the physical consequences of making some variables go to zero or infinity.

When students reach the final result in problem solving, they quite often believe that they are done. Clearly against this belief, in several moments of the course the professor emphasized the importance of "playing with the final result" in order to "see the physics behind it." In fact, this is one of the core skills related to the structural role of mathematics in physics. As Hertz once said, mathematical formulas are "wiser than we are" and we often "get more out of them than was originally put into them" ([2], p. 13). The fact that we are able to find more physics through the interpretation of mathematical expressions testifies that mathematics is not merely a language that offers a precise description of physical phenomena, but that in many cases the mathematical formalism guides the physical thought.

The first two categories allow the identification of specific translation or transfer moments, i.e., going from physics to mathematics and vice versa. Both mathematizing and interpreting are among the abilities that characterize what it means to "think like a physicist" (see mathematical sophistication in [23]). They are essential structural skills that are constantly being emphasized in the professor's didactic discourse. However, in many situations, to "act like a physicist" involves using mathematics as a mere instrumental tool, and the following category locates moments in which this approach was identified in the lectures.

\section{Technical}

When watching the lectures, the role played by an instrumental use of mathematics (technical dimension) in the professor's didactic discourse was an important focus of interest. In fact, physicists often treat mathematics as a set of practical rules that provide them with a safe reasoning guide. It is actually quite useful for physicists to allow themselves to be "led by the safety of the formalism," since it is not necessary to prove the validity of every mathematical step. Differentiation or integration rules, mathematical theorems (Pythagoras, Gauss, Stokes, etc.), trigonometric identities, among many other examples, are extremely useful tools for professional practice in physics. But what role should such a technical approach play in physics lectures?

This category was thought to answer this question in the context of this case study. It locates moments of the lectures when the technical use is made both within the context of physics-e.g., using arguments of authority, simply quoting equations - and when no connection with the physical world is made and the professor is doing "just math." When the approach is exclusively instrumental, it is categorized as $\mathrm{T} 1$ - technical procedural. Here are two examples:

This is a function of $x$ and $y_{\max }$ is given by the equation of this line here. What is the equation of the line? I know that $y_{\max }$ should be given by the function $y=\alpha x+\beta$. [...] Fellows, in physics we have no self-affirmation problem with mathematics. So if you want to look for that at a table, just do it. We want the quickest way. (lecture 6-51:20-52:40).

Once you have done that, you are allowed to turn the mathematical crank. Just throw the values there and start manipulating. (lecture 9-44:40-45:00).

After watching several lectures it became rather easy to identify moments to be categorized as technical procedural (T1), since the professor's body language usually indicated a careless attitude, which was evident when he performed algebraic manipulations quickly and often turned his back to the class. In several occasions during these manipulations, students pointed out mistakes and the professor quickly accepted their corrections, showing an attitude of indifference.

At certain occasions, he behaves like a mathematics lecturer and provides (mathematical) justifications for the validity of properties, rules, and theorems, as 
exemplified in the following. These moments belong to a subcategory of the technical dimension (T2 - technical conceptual).

\section{Student asks: This r-squared is actually not a vector, right?}

Professor: What is a vector squared? It is $r$ dot $r$, right? Is that clear to you? [...] Imagine a vector $r$ with its components $x$ y $z$. If you calculate $r$ dot $r$, it gives $x^{2}+y^{2}+z^{2}$, which is equal to the square of the vector's magnitude. (lesson 9-13:20-15:00).

Besides identifying moments of a sole technical approach, it is crucial to determine their duration and especially their location in the whole unit of a lecture or an episode, especially considering the possibility of conducting comparative studies. In fact, in this case study the Videograph timelines show that this technical modus operandi rarely appears alone. The most common situation is to find the instrumental approach occurring between moments of mathematization and interpretation. This is exemplified by the analysis of a problem-solving episode in Sec. V as well as in the timelines presented in the Appendix.

\section{B. Second dimension: Pedagogic and linguistic strategies}

The next three categories refer to specific strategies used by the professor when focusing on translation processes (i.e., mathematizing and interpreting). In general, his extensive use of visual representations and analogies is quite noticeable, especially when new concepts are introduced.

\section{Visual representations}

When representing physical situations mathematically (M-mathematizing) or interpreting mathematical expressions physically (I-interpreting), the professor's didactic discourse was remarkably visual. As the lectures were being watched, it was noticeable that semiotic resources such as gestures and pictorial representations (drawings, charts, diagrams, etc.) played a central role in his explanations, which influenced the choice of categorizing such moments. In the interview, the professor justifies this intensive use of visual representations by saying that "physical knowledge is silent" and cannot be fully expressed by words.

This insufficiency is supported, for example, by the work of the American physicist and linguist Lemke [48]. After analyzing several scientific articles, the author states the following:
Science is not done, is not communicated, through verbal language alone. It cannot be. The "concepts" of science are not verbal concepts, though they have verbal components. They are semiotic hybrids, simultaneously and essentially verbal-typological and mathematical-graphical-operational-topological. [...] To do science, to talk science, to read and write science it is necessary to juggle and combine in canonical ways verbal discourse, mathematical expression, graphicalvisual representation, and motor operations in the natural world. ([48], p. 89, my emphasis).

Thus, by establishing a category for the moments when visual representations played an essential role in the professor's discourse, it is possible to recognize the "multimediatic" character of the meaning making process. These visual representations were divided into two subcategories, namely, V1-gestures and V2-pictorial, and some examples are given below.

The mathematical object appropriate to describe things that do this [makes a circular motion with the finger] is the curl. And this here [points to the divergence] is the mathematical object used to describe things that do this [spreads his arms] (lecture 24-1:45:00-1:45:20).

If you take a surface like this [bends a sheet of paper], for example. Below here [gestures] passes a lot of charge, but above here [gestures] almost nothing. If you have a wire, then $i$ [current] is enough, but if not, then $\vec{j}$ [current density vector] is more accurate. (lecture 26-1:11:401:12:00).

This type of discourse supported by visual resources was mostly found when new concepts were being introduced and mathematized. It appeared to complement (or even expand) the traditional definitions expressed in words or equations. In this sense, the use of multiple semiotic resources is possibly related to the quality of the professor's explanations. In fact, the analysis of teaching episodes showed that the focus on the structural dimension (mathematizing and interpreting) often demanded a more "multimediatic" discourse. This seems to find resonance in Lemke's work, particularly when he talks about the notion of meaning multiplication:

[...] the principle of functional cross-multiplication in multimedia genres shows us how we can mean more, mean new kinds of meanings never before meant and not otherwise mean-able, when this process occurs both within and across different semiotic modalities (i.e., language, visual representation, mathematics, etc.). ([48], p. 94, my emphasis). 


\section{Analogies}

Another very important explanatory resource related to the structural dimension (especially mathematizing) is the use of analogical reasoning. Throughout the course, the professor makes use of a wide range of analogies and metaphors. In most cases, this was done by means of presenting everyday life situations and comparing physical phenomena. At other times, formal similarities were highlighted and unifying mathematical structures were made evident. This distinction led to the choice of two subcategories [49] to analyze the use of analogical reasoning in the lectures.

A1-Material analogies (everyday life examples and metaphors).-When a new concept is introduced, the professor often uses a vast repertoire of everyday life situations and analogies between physical phenomena as exemplified here:

Can dogs calculate the gradient? Yes, they can. Put a dog in the corner of a dark room. Then pick up a tasty steak and put it here [points to the opposite corner of the room]. [...] What does the dog do? It lifts its nose and smells. After one sniff, does it know where the steak is? No! With one sniff it knows that there is a steak, okay? Where is it then? Now it has to calculate the gradient. It turns its head randomly. And then what will it measure? It measures the direction in which the smell increases, okay? Then it walks a little in this direction, stops and sniffs again. And then, by repeating this process, it finds the steak. In other words, it calculates the gradient. (lecture 19-14:20-17:40).

This excerpt reflects a frequent attitude to seek for concrete examples in order to offer the students an arsenal of complementary images with which they can identify. It is also clear that in the process of building a theoretical concept, the professor chooses a from-concrete-to-abstract approach. This identification with real life situations and experiences seems to be a crucial condition for a meaningful mathematization of physical situations. The strong conviction of this importance was clearly identified during the interview with the professor.

A2-Formal analogies (common mathematical structures).-According to Hesse ([50], p. 202), "an analogy in physics is a relationship between two hypotheses, or between a hypothesis and certain experimental results, in which certain aspects of both relata can be described by the same mathematical formalism." Similarly, Feynman stresses that "the equations for many different physical situations have exactly the same appearance" ([51], 12-1). The explicit mention of formal analogies was categorized as A2 and is exemplified in the following.

The required mathematics is already done. This law here [Ampère-Maxwell law] although we have not seen its physical meaning yet, it is, from the mathematical point of view, similar to this [Faraday's law]. So if you understand the math of this one [Faraday] then you understand the math of this other [Ampère-Maxwell]. (lecture 24-31:20-32:00).

Analogical reasoning played an essential role in the development of the electromagnetic theory (see, for instance, the original works of Thomson and Maxwell). The very words flux, tension, or current reflect the significance of using formal structures developed in a concrete or mechanical context to reason about the unknown or unseen electromagnetic phenomena. Throughout the course, the professor explicitly mentions the importance of analogical reasoning for physics and dedicates considerable effort to developing this reasoning skill with his students.

\section{Deduction}

The notion of proof is central for mathematics. Its main idea is to start from an "evident" set of axioms and, by logical deductions, prove the veracity of propositions. This style of reasoning is in the core of the epistemological notion of physical explanation [52] and is widely found in several physics masterpieces, such as Newton's Principia and Einstein's work on special relativity. In fact, Einstein explicitly mentioned the similarity between geometry and theoretical physics by saying the following:

The theorist's method involves his using as his foundation general postulates or "principles" from which he can deduce conclusions. His work thus falls into two parts. He must first discover his principles and then draw the conclusions that follow from them ([53], p. 110, our emphasis).

Being able to comprehend logical derivations of formulas allows one to recognize how physical assumptions, such as the principles of minimal action or energy conservation, are imposed by physics instead of emerging from mathematical manipulations. The ability to separate physical assumptions from mathematical manipulations is strongly related to a meaningful understanding of the role of mathematical reasoning in physics (structural dimension). Thus, this category was designed to locate moments where deductive reasoning was the focus of the lectures. Although much less common, this approach is found in some crucial discussions, as in the following passage.

[Discussion after problem solving-Determine the electric flux through a disk caused by a point charge] This happened because I used that expression [Coulomb's law] and it falls with $1 / r^{2}$, ok? What is happening is the following: the field drops with the square of the distance, but the surface increases with the square of the distance, so 
that the product of the surface times the field remains the same. [...] What is the idea of Gauss's law? The idea is to invert the logical order of these two statements. And it raises the flux constancy to a greater truth than that [Coulomb's law]. So now we see an inversion of values. We started believing in this [Coulomb's law], which is an empirical fact. But what physics decided to say that this [flux constancy] is more important. And if I say that this is more important, what do I learn? I can now understand why the field drops with $1 / r^{2}$. [...] We can now derive Coulomb's law from Gauss's. We can show where the $4 \pi$ comes from. (lecture 12-1:41:20-1:42:40).

The emphasis on the words show and derive are evidences that the deductive aspect of physics is being approached. The greater generality of Gauss's law is expressed when the professor mentions that it can be used to derive Coulomb's law and to show where the "mysterious" $4 \pi$ comes from. This emphasis on deductive reasoning is normally found at the end of topics or chapters and has the goal of highlighting big syntheses. It is especially present when the professor stresses the power of the theoretical edifice represented by Maxwell's equations.

\section{Third dimension: "Stops" dedicated to metalevel reflections}

The last set of categories address specific moments in which a clear interruption of the "normal course" of the lecture is identified. Another way to interpret this dimension is to imagine a lecture as train travel and treat the moments described by the following categories as stops made along the way to admire the view [54]. These stops were generated mainly by philosophical discussions, metacognitive remarks, or questions posed by the students.

\section{Philosophy}

Discussions about the nature of physical knowledge were quite common during the lectures. This focus is possibly explained by the professor's background, which includes studies in philosophy of physics. In many instances of the lectures - often during the first 20 minutes or when a student raised a question-the professor thematizes metatheoretical aspects of physics, such as "What is a theory?" "What is the relation of mathematics to physics?" "What is the relationship between theory and experiment?," "How do we model reality?," among others. Because of the high frequency and wide variety of this approach, it is impossible to reproduce the discussions in depth here. The following fragments provide examples of passages identified in this category.

This frame of reference is a reasoning instrument. It is not in nature, but in your mind. It is an invention. (lecture 5-1:00:20-1:00:40).
What is the relation between mathematics and physics? This is a very complex relationship. [...] In physics, we use mathematics as a reasoning instrument to think about the world. [...] But we use math in quite an intuitive way. Of course the mathematical rigor is important, but when we work in physics we do not worry about it so much. [...] Our way of thinking is connected to the material world. Several mathematical theorems were intuited by physicists, they did not demonstrate them. Stokes' theorem is a good example. (lecture 6-1:20-6:00).

A great number of other examples could be given, since the professor's philosophical discourse is both very wide ranging and deep. The implicit message, which was confirmed in the interviews, is the following: the student should not only be able to do physics, but also to reflect on what it means to do physics. This kind of approach is not usually found in traditional physics lectures and tends to be delegated to specific "history and philosophy of physics" courses. The examples found in this electromagnetism course as well as the interviews with the professor show that it is possible (even desirable) to do physics and to think about doing physics at the same time. In fact, the quality and depth of the discussions highlight the benefits of conducting philosophical discussions from an insider perspective.

\section{Metacognition}

Thinking about one's own thinking has proven to be an important habit of mind for good problem solvers. Moreover, research on cognitive psychology has shown that metacognition is a powerful predictor of learning outcomes [55]. During the lectures, a constant intention of the professor to encourage this kind of metacognitive reflection was perceived. At numerous times he paused the lecture with metacognitive remarks such as, "Now ask yourself if you truly understood this," "Do not think this is easy?", "We usually need a lot of time to understand this," among others. These moments were categorized as metacognition and seem to be another important feature of the lectures. The overall perception is that the professor is fully aware of the difficulties he faced in understanding the abstract concepts of electromagnetism and makes them explicit to students, as opposed to acting as if everything were easy and obvious. This attitude of encouraging metacognition is exemplified in the following excerpts:

This may look simple, but if you make a small mistake here, you ruin everything. So you need to be careful. [...] This is serious! You won't be able to solve the problem this way. In general, people who have difficulty in connecting the symbols during the calculations begin to solve a problem and do not realize when the problem 
is over. For them, the problem is to calculate something, and then they start to calculate, calculate, calculate... The answer is already found, but they continue to calculate, calculate, calculate. [Laughs] We cannot work this way. (lecture 9-42:40-44:00).
It is very nice that he asked this question. His question indicates a certain misunderstanding. But this confusion occurs with everyone who studies this topic for the first time. And if it still did not happen to you, it is because you have not realized it yet. It is taking place in your

TABLE II. Summary of the categorization system.

\begin{tabular}{lcc}
\hline \hline Category & Description & Example \\
\hline
\end{tabular}

First: Translation and manipulation

Mathematizing M2-structures

M1-assumptions

Interpreting (I)

Technical T2 - conceptual
mathematics

T1-manipulation and authority

Second: Linguistic and didactic strategies Visual V2-pictorial V1-gestures Analogies A2-formal

A1-material

Deduction (D)

Third: Metalevel stops Philosophy (P)

Metacognition (Met)

Questions (Q)
Mathematical structures are used to represent physical quantities and their relationships. Essential aspects are identified and physical justifications are offered.

Idealizations, approximations, and selection of relevant variables are addressed explicitly.

Mathematical expressions are interpreted physically. Special cases are commonly used.

Conceptual explanations to mathematical rules and procedures are given. The justification is solely mathematical.

Technical manipulations are performed, equations are blindly used, and arguments of authority are evoked. Careless posture.

Drawings, diagrams, and other pictorial representations are essential explanation sources.

Gestures play an essential explanatory role.

Formal similarities and differences are highlighted. Unifying character of mathematical structures is mentioned explicitly.

Everyday life situations, analogies, and metaphors are used to give meaning to abstract concepts.

Aspects of the logical-deductive character of physical knowledge are mentioned. Formulas are deduced from physical principles.

Philosophical discussions are conducted. Various aspects of the nature of physics are thematized.

Students are encouraged to reflect on their own thoughts. Difficulties in understanding abstract concepts are made explicit.

Questions related to the interplay between physics and mathematics are raised by students. Long time is dedicated to the explanation.
The problem is that $i$ [current] is a scalar. In order to be more precise, we need a vector $[\vec{j}$, current density].

This paper sheet is not bidimensional, but I can think of it as if it were.

What does it mean to integrate over dy? [...] What does this expression say? [...] What if $x, y$ or $z=0$ ?

Imagine any vector $r$ with components $x$, $y$, and $z$. If you calculate $r$ dot $r$ it gives $x^{2}+y^{2}+z^{2}$, which is the square of the vector's magnitude.

Now you are authorized to turn the mathematical crank. Throw it there and start manipulating.

The positive charge creates a yellow field [draws]. The negative creates an orange field [draws].

This is theta [points at the door], this is theta plus $d$ theta [opens the door]

They took this mathematical formulation and used in different situations. [...] But there are also important differences.

Can dogs calculate the gradient? Yes. [...] How can it find a steak inside a dark room?

We can now derive Coulomb's law from Gauss's. We can show where the $4 \pi$ comes from.

In physics we use math in quite an intuitive way. Our way of thinking is closely connected to the material world.

This confusion occurs with everyone who studies this topic. It is taking place in your mind right now, but you are not aware of that.

Student: There is " $g$ " in the result, shouldn't it be " $v$ "?

Professor: It is really great that you asked that. 
mind right now, but you are not aware of that. (lecture 12-37:00-37:40).

The focus on regulating thought processes and recognizing complexities is evident in the professor's metacognitive discourse. His attitude contrasts with the usual posture of presenting physical knowledge as linear, logical, and even trivial, which implicitly suggests to students that they should not experience difficulties when trying to learn physics.

\section{Questions}

Even though the lectures of this case study can be described as quite traditional, the professor's Socratic discourse seems to create an atmosphere in which questions are very welcome. In the interviews, he clearly states his appreciation for questions and his desire to encourage students to ask them frequently. In fact, the analysis of the lectures showed that a lot of time and effort is dedicated to each answer. Moreover, a positive reaction is commonly identified in his answers (e.g., "this is a very good question," "you are right"). Thus, this category was created to locate moments in which the professor interrupts the course of a lecture due to questions posed by students. Two examples of questions related to the role of mathematics, which generated long explanations, are given in the following:

Student: The flux has to be in 3D, right?

Professor: Good question. How many dimensions does the flux have? [...] Let's go back to the original problem. What is flux? In the case of water or bees, you will count what passes through a surface. But now consider a street and count the number of cars passing on the street. [...] How many dimensions does the flux in the street have? [...] Two, ok? Cars on a street is a two-dimensional problem, right? It is not like bees or air, they are flying all over the place. You do not have a car in the third dimension. [...] So the movement of the car in the street can be considered twodimensional. And then the flux will be through what? A line. The surface is not important. You count on a line. (lecture 12-31:40-34:00)

Student: Isn't it easier just to throw everything in the equation and then reason about the problem's geometry?

Professor: But how will you throw everything in the equation without knowing what $\vec{r}_{p}$ and $\vec{r}_{q}$ are?

Student: You work with them like vectors; symbolic. Professor: But what does the symbol stand for?
Student: Can't I just put $\vec{r}_{p}$ minus $\vec{r}_{q}$ over [...] and then this minus $[\ldots]$ ?

Professor: You can, but if you do not draw a picture, you won't know what you are talking about. And this is the problem. $\vec{r}_{p}$ is $\vec{r}_{q}$ but you cannot find out what $\vec{r}_{q}$ is. Your formalism is not deeply connected to the problem. (lecture 9-36:00-37:00)

Every physics teacher would probably say that questions are welcome in their class. However, students are easily capable of sensing this appreciation in the way teachers react to questions. In the lectures of this case study, there are many examples of questions motivated by students' struggle to understand the interplay between mathematics and physics. Being able to create an atmosphere where students feel confident to ask questions seems to be crucial for a successful teaching of physics, especially concerning the learning of the structural role of mathematics.

\section{Categories summary}

The nine categories presented and illustrated in this section constitute the basis for the analysis of the teaching episodes using the software Videograph. Table II contains a summary of the categorization system.

\section{ANALYZING TEACHING EPISODES}

The categories presented and exemplified in the previous section are used to analyze teaching episodes extracted from the lectures. The coding process (see Fig. 1) consists in dividing an episode in 20-s time units and assigning to each unit one (or more) category. The final result is a timeline, which can be seen as a kind of $\mathrm{x}$ ray or spectrum of the episode. This visual representation gives an idea of the duration of each category and the order in which they appeared in the lectures. Even though such visualization provides a dynamic image of what happened during an episode, its combination with the transcripts is essential for a deeper understanding of the professor's didactic discourse.

Nineteen episodes were selected for a deep analysis in this case study. These were divided into two groups, namely, the ones dedicated to the introduction or explanation of a particular concept and the ones focused on problem solving. In order to provide the reader with a broad picture of the categorization process, two episodes (one concept and one problem) are analyzed in the following. In the Appendix, the timelines of six other episodes, including a brief description of their essential features, are presented. The complete analysis of all 19 episodes can be found in Ref. [42].

\section{A. Episode 1: Flux of a vector field (concept)}

Lecture 12-Duration: $30 \mathrm{~min}$.

The concept flux of a vector field is present in all four Maxwell equations and, therefore, is of fundamental 


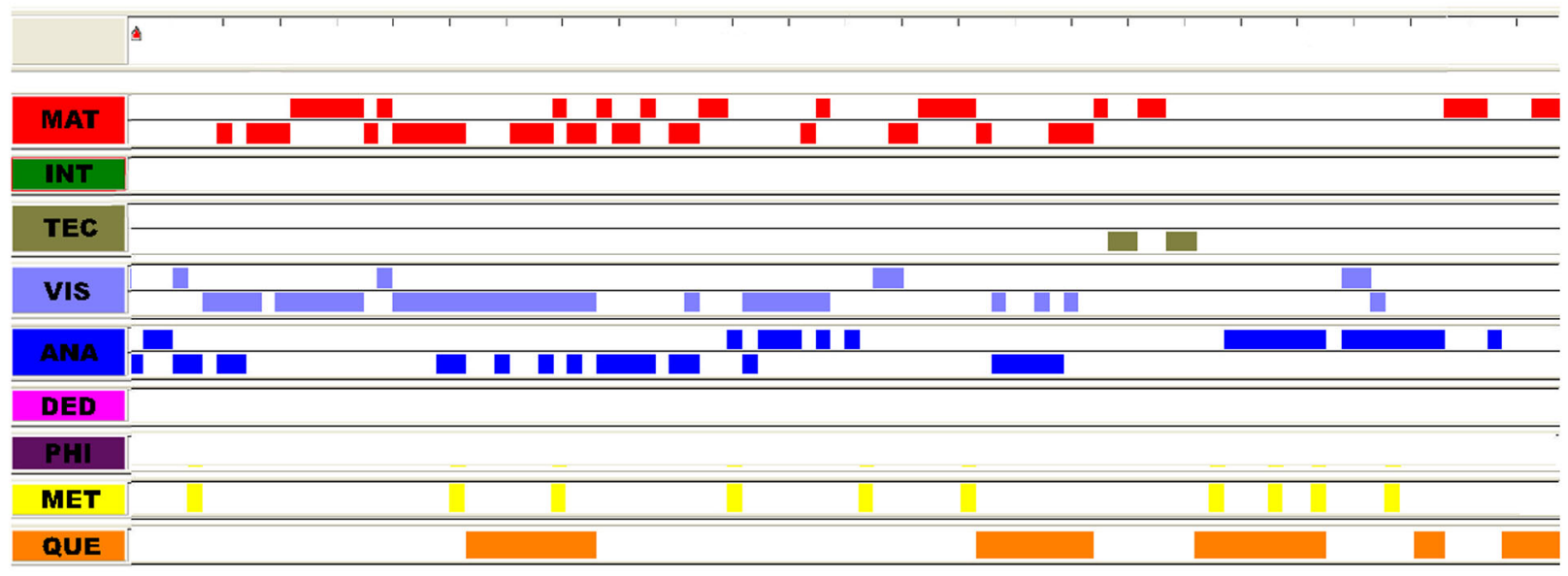

FIG. 2. Timeline of the flux episode.

importance for the understanding of electromagnetism. Moreover, previous studies [56] have already shown that students encounter difficulties with it and often mix the concepts of field and flux. These are among the reasons for selecting this episode to exemplify the categorization of a teaching episode dedicated to the introduction of a concept. As its timeline (Fig. 2) indicates, this episode is focused on the mathematization of the flux concept. It is also evident that several visual representations and analogies are used in this process. Furthermore, it is possible to identify some metacognitive stops and long answers to students' questions.

The episode starts with the professor's survey of the students' intuitive notion of flux. He gradually identifies relevant variables for the mathematic description of this concept:

01:40-04:00 How do we think about something that flows? [...] There is no notion of flow unless you support a mathematical surface on the door. Thus, we can only speak of flux through a surface. [...] [turns the book parallel to the plane of the door] Is there air flowing through this surface now? Yes, okay? [turns the book] It changes, right? So the notion of flux depends on how you orient the surface. From the mathematical point of view, we have to take this into account. (M1, V1, Al).

Afterwards, the search for an appropriate mathematical representation is gradually approached:

04:20-06:00 Ok, we have to project something. What is the light flux from the lamp through this surface here? [...] One important remark: Flux is the end result. How am I going to say mathematically that there is flux when the surface is like this [perpendicular], and not when it is like this [parallel].

Student 1: It has to be perpendicular to the flux.
Student 2: Put some angle there.

Professor: We cannot say "perpendicular to the flux" because flux is the result of our calculation. $O k$, it has to be perpendicular to something. What is the relevant magnitude? [...] This vector and what from light? [...] Its direction, ok? How do we quantify that? Which vector enters in our calculation? (M2, V1, Al)

In these fragments we observe various strategies used by the professor to highlight the need of a surface to think about flux and its dependence on direction. Instead of starting by mentioning a formula, it is clear that the goal is to mathematize, i.e., build a mathematical representation of an idea extracted from the world. In this process it is noticeable that idealizations are explicitly mentioned, relevant variables selected, and appropriate mathematical structures (vectors, dot product, integral) chosen.

Similarly to other moments when new concepts were introduced, numerous analogies are used during the flux episode:

01:40-02:00 What is water flow? How do we think about this situation? Think of a river. Imagine that you have a river here [draws] and the water is flowing. (M1, A1, V2).

09:00-09:20 What is the flux of light from the lamp through that surface here? (MI, Al, Vl).

10:40-11:00 Imagine a bee swarm coming through that door. Is there a flux of bees through this surface? (M1, Al, V1).

14:40-15:00 Can we speak about the flux of $g$ [gravity] through this ds? (A2, VI). 
In addition to metaphors and material analogies with other areas of physics, the frequent presence of formal analogies is also evident in this episode. This is seen when the professor points out that the same mathematical structure can be used to represent different phenomena.

01:20-01:40 This vector can be anything, it is a generic $\vec{F}$. It can be an electric field, magnetic, velocity, gravitational field, it can be anything. (A2).

Aside from approaching similarities, highlighting differences is also very important when addressing formal analogies. In the particular case of the flux concept, one crucial problem arises due to the usual meaning attached to the word flux (or flow), which implies the notion of movement, i.e., something is moving (or flowing) across a surface. However, when it comes to the flux of the electric or gravitational field, there is no notion of movement involved. Aware of the fact that this is a common learning difficulty for the students, the professor explicitly addresses this crucial difference when he calculates the flux of the gravitational field:

14:40-16:00 Can we speak about the flux $g$ [gravity] through this $d s$ [surface element]? In fact we can and we do that. But then the word flux is not a good one. Because when I talk about velocity, the bee swarm for example, the bee was here and it crossed to the other side of the section. The bee really went through the mathematical surface. So when I speak about the flux of a velocity field, the word flux is good because it contains the idea of something moving. Is the vector $\mathbf{g}$ passing through the surface? No. The $\mathbf{g}$ is standing here. [...] In this case the notion of movement does not exist in the word flux. But we use the word anyway. We do talk about flux of gravitational field. (A2, V1).
Even though the lecture is part of the electromagnetism course, the professor does not explicitly mention the electric flux in this episode. In fact, the first remark about electric flux comes from a student in the end of this episode, probably realizing the analogy with the gravitational field:

\section{8:20-30:20 Professor: Is there a Gauss's law for} gravitation? [...] Yes. Consider the Earth here. There is $g$ everywhere [draws radial field lines]. Then you take a mathematical surface. [...] What is the flux of $g$ through this surface? And what Gauss's law says is that it is equal to the mass of the Earth. [...] Again, $g$ is not flowing through the table.

Student: The electric field will also be like this, it is not moving. It will be just there.

Professor: Exactly! The same thing for the electric field. And what will Gauss's law say? What is the analogous to mass in the electrostatic case? The charge. (A2, V1, Q)

This episode highlights the multiple resources used by this professor to focus on the structural role of mathematics. The emphasis is noticeably on mathematizing the flux concept and, for this purpose, analogies (both material and formal) and visual representations (gestures and pictorial) are used extensively. Metacognitive remarks and answers to students' questions are also frequently found throughout the episode. It is worth noting that similar profiles were found in episodes where other concepts like charge density and electric current were introduced or mathematized (see the Appendix). Using the analytic tool presented in this work to categorize other lectures could reveal important

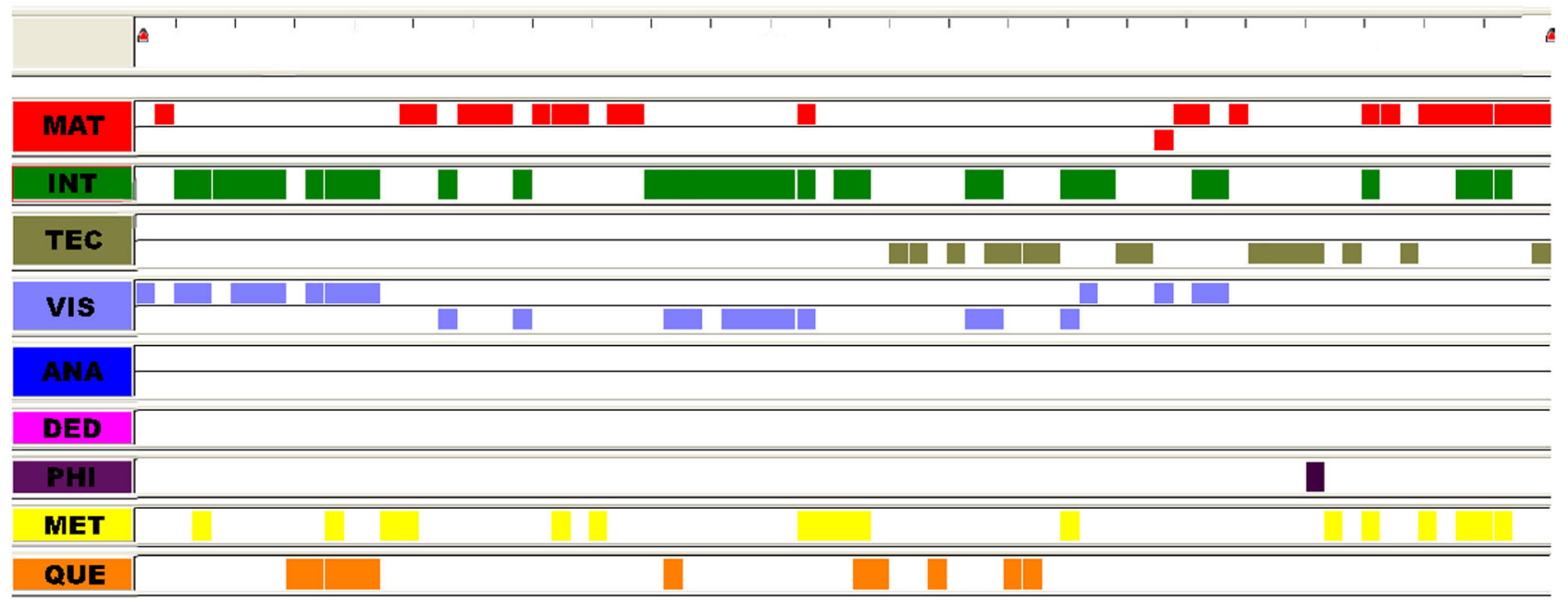

FIG. 3. Timeline of the charged plate problem episode. 
differences. In a didactic approach focused on the technical role of mathematics, for instance, the electric flux equation would likely be presented right at the beginning and followed by problem solving. In general, this would involve a more rational and straightforward approach as well as a less varied (i.e., less colorful) spectrum.

\section{B. Episode 2: Net charge of a plate (problem)}

Lecture 6-Length: 25 min.

Problem: Calculate the net charge of a rectangular plate with sides $D$ (horizontal) and $C$ (vertical), whose surface charge density is given by $\sigma=(a x+b) y$.

To solve this problem in a purely procedural (technical) way would require 5 minutes at most. Why did the professor spend 25 minutes of the lecture with its solution? The timeline (Fig. 3) provides some hints. It reveals a strong presence of the category interpretation (green), which is related to the physical meaning of representing surface charge density by mathematical functions $[\sigma(x, y)]$ and integrating functions to obtain the net charge. Gestures and figures are heavily used as explanatory resources. It is also noticeable that, at various times, remarks of a metacognitive nature (yellow in Fig. 3) are made with the aim of encouraging reflection on cognitive processes during resolution. In addition, portions of mathematization and technical manipulations are also identified.

The problem is proposed as follows:

01:20-03:00 Imagine a rectangular plate [draws] charged with the function $\sigma=a x+b$. If you had to make a drawing of the charge contained in this plate, how would it look like? [...] One possibility is to draw points to show regions with different densities [points closer or further away from each other]. Here the charge is weaker. As you go farther away the charge becomes more concentrated [the bigger the distance from the origin, the closer the points]. What is important here is to look at this function and know what it represents in nature. [...] Another possible function is: $\sigma=(a x+b) y$. How does the charge distribution look like now? [...] It varies in two directions [draws], it grows here [moves to the right] and also in this direction [moves up, vertical]. Each function represents a particular charge distribution. (M2, I, V2, Met).

The question of a student indicates that he has difficulty interpreting the meaning of a function that does not depend on $y$ and it motivates a more detailed explanation.

04:20-06:00 Student: In the first case there is no dimension in $y$, right?

Professor: Yeah, what does that mean?

Student: It means that it has a linear growth.

Professor: Let's consider his question, what does it mean not to depend on y? For example, consider this band [vertical]. If you take this band and cut it up like that. All the pieces have the same size [draws small rectangles on the same vertical band]. Where does it have more charge, here [bottom] or here [top]?

\section{Student: The same}

Professor: Yes, so this is what is being said by the function. Now if you compare this [vertical band closer to the origin] with this [farther away to the right], this one [latter] has a greater charge. In the other case $[\sigma=(a x+b) y]$ [draws horizontal and vertical lines], in this square [top right] there is more charge than here [bottom left]. I am taking equal areas to make our lives easier. The charge increases in this direction [up] and also here [to the right], right? (I, V2, Met, $Q$ )

The charge element is represented by $d^{2} q=\sigma d x d y$ and the total charge by the integral $\int_{0}^{D} d x \int_{0}^{C} d y \sigma$. After this mathematization step, the focus is again directed to interpreting the meaning of each integral operation, which is done with the help of a sheet of paper.

10:40-12:40 What does it mean to integrate over dy? That I am adding all the charge contained on this band [points to vertical band on drawing]. The result of the operation [charge] is a number. Then, to integrate over y is equivalent to do this [folds a sheet of paper turning it into a horizontal bar]. After we integrated over dy, the $y$ dimension is gone. And what is left is a bar. And then what does it mean to integrate over $d x$ after that? Is to do that [crumples the paper stripe turning it into a "ball"]. And that is the total charge. (I, VI, Met).

Calculus is essential for virtually all branches of physics. In order to solve problems, students quite often have to know which integration technique can be applied to a particular function. This can make them reflect less on what the calculations represent physically and more on how to solve the integrals (technical dimension). In this passage, the greatest importance is given to the interpretation of the meaning of the integration, which is done with the help of gestures. Saying that to integrate over $x$ and then over $y$ means that you first transform the sheet of paper into a bar and then into a ball provides a physical or visual meaning to the operations. This attitude reinforces his emphasis on the structural dimension of mathematics.

Nevertheless, this does not mean that the technical part of mathematics is not approached. In this episode it is possible to find moments when the "mathematical crank" was turned to solve the integrals as shown by Fig. 3. It is easy to notice a considerable change in the professor's posture when performing these calculations, which denotes a somewhat careless attitude. Moreover, it is also noticeable 
that such technical approaches are usually followed by interpretation phases.

A small change in the problem allows the professor to better exploit its potential and provides another possibility to emphasize the physical meaning of mathematical representations. The plate is now triangular (the rectangle basis is maintained, but a triangle is constructed by drawing a diagonal and eliminating the superior triangle). In the following passages it is quite difficult to distinguish between mathematization and interpretation:

19:20-20:00 What changes in the calculation if I have half a rectangle now? In other words: Where, in this calculation, does the information that the body is a triangle and no longer a rectangle enter? (MI, VI).

23:00-25:40 Where does the information about the shape of the body go? [...] In the first example I integrated the variable y from zero to $C$. Why? Because the body was like this [rectangle] and I wanted to get from here to there [0 to C]. For each point the distance $y$ was constant. This changes now. Now for each $x, y$ has a maximum value $\left[\mathrm{y}_{\max }\right]$ and I am going to take this away [erases the upper limit of the integral] and put $y_{\max } u p$ here, given by that function $\left[\mathrm{y}_{\max }=-(\mathrm{C} / \mathrm{D}) \mathrm{x}+\mathrm{C}\right]$ [draws a straight line from $\mathrm{C}$ top left- to D-bottom right]. [...] What do we need to pay attention to? The shape of the object is given by the limits of integration. [...] Is the total charge different? Why is it different? How does the calculation know that? [...] The expression has to be informed of what you are doing. Then at each step of the calculation, you have to put some information there. Each information enters in one particular place. The information about the density goes here [points at the $\sigma]$. The information about the shape goes up here [limits of integration]. (M2, I, T, V1, Met).

At the same time that the professor is mathematizing (e.g., How do we express mathematically the fact that we now have a triangle?), he is interpreting the meaning of mathematical operations. This almost simultaneous approach is highlighted in the categorization of the last 3 minutes of this episode. The kinds of (unusual) questions posed by the professor during the process of solving this problem-such as how does this information enter the calculation-attests to his focus on the structural dimension of the relationship between physics and mathematics. Moreover, the explicit monitoring of the solving process (metacognition) is stressed in his discourse.

Like in the flux episode, one promising strategy would be to compare the solution of the same problem conducted by other lecturers and use the analytical tool to identify core differences. Again, one approach focused on the technical dimension would likely be more straightforward, contain fewer categories (less colorful timelines), and last less time. Overall, the number of problems solved throughout the electromagnetism course is quite low. However, a great amount of time was dedicated to their formulation, solution, and discussion, as exemplified in this episode (the timelines of the other three problems are provided in the Appendix). This indicates that the professor does not regard problem solving as an application of definitions and procedures (technical dimension), but as valuable opportunities to learn how to use mathematical reasoning to structure physical situations (structural dimension).

\section{CONCLUSIONS AND RESEARCH PERSPECTIVES}

How to facilitate students' understanding of abstract concepts is certainly a major concern of every dedicated physics teacher. However, specific guidelines on how to be successful in this task are not always available. With the goal of contributing to the research (and practice) in this field, I have analyzed the electromagnetism course given by a highly appreciated and experienced physics professor at the introductory level. The focus was directed to the way mathematical reasoning was used in his didactic discourse. Considering an overall distinction between a technical and a structural role of mathematics, special attention was given to the strategies used by the professor to teach the students to use mathematics as a reasoning instrument to frame the physical world. A coding process developed with the software Videograph for the analysis of teaching episodes was presented and exemplified.

For organizational purposes, the categories were divided into three subsets that address different, yet complementary, dimensions of the lectures. The first dimension refers to how mathematics is treated and encompasses both translation processes (mathematization and interpretation) and the technical approach. In general, the analysis shows that the professor dedicates a considerable amount of time and effort to build mathematical representations of physical concepts. This was done with an explicit mention of idealizations or abstractions (M1) and clear justifications for the use of specific mathematical structures (M2). In fact, mathematization (as a process) can be easily disregarded in physics teaching, for instance, when formulas are prematurely presented and used as calculation tools (technical approach). The didactical choices of this professor stress his emphasis on a gradual and meaningful construction of mathematical representations in physics teaching, which indicates his focus on the structural role of mathematics.

After obtaining a mathematical expression, either for representing a physical concept or as the solution of a problem, the focus was immediately directed to the interpretation of its meaning (I). For this purpose, special and/or limit cases were frequently discussed. Specific questions highlight this approach, such as "How and where does this information enter the calculation?" "Which term 
is responsible for this physical property?" "What is the physical implication of the fact that the expression depends (or not) on this factor?" His reason for focusing on interpretation is expressed in one interview: "The final result/equation is like a finger. It is pointing you to something really interesting. If you stop there, you leave the most important and fascinating thing out".

Thus, concerning the first dimension, the main focus of the lectures was clearly on translation processes (mathematical sophistication [23]), which belong essentially to the structural approach (see Table I). Although technical manipulations $(\mathrm{T})$ were also commonly found, they never appeared alone and were almost always preceded by mathematization and followed by interpretation. Moreover, the technical approach was easily identified by a careless attitude of the professor. When asked to justify this posture, he said, "This is sort of a political attitude. I want to show that this is part of physics, but it is far from being everything. I don't want them to think that this is the most important, which is a widespread view in physics instruction."

The second dimension concerns particular strategies used by the professor when mathematizing and interpreting. Among them, visual representations (V1 and V2) were used thoroughly as linguistic resources to make meaning, which reinforces Lemke's [48] argument that scientific concepts are "semiotic hybrids." Furthermore, mostly at the end of chapters, the deductive reasoning (D) was highlighted with the goal of underlining big theoretical syntheses. This was particularly evident when the predictive power of Maxwell's equations is emphasized.

When introducing a new concept (e.g., charge density, electric flux, electric current), a from-concrete-to-abstract approach was clearly preferred, which was manifested in his extensive use of rather unusual analogies with everyday life situations (A1). In one interview, the professor explains his didactical choice by exploring the analogy between calculating the gradient and a dog searching for a steak in a dark room (Sec. IV): "The example of the dog is very good and the students understand it. [...] We have a direct experience with dogs. [...] The relation a dog has with the smell of a steak is very strong; it knows how to find it, even without knowing how to calculate the gradient. So this means that the notion of the gradient was extracted from the world. The gradient is a human construction, it does not belong to the world; the world came first. [...] So, the persons who invented this mathematics took this property out of the world and translated it into mathematical language. Then, if you are not able to find this deep connection with the world, this becomes just a language, which is somehow meaningless to you".

The last three categories describe moments of the lectures in which a clear interruption of their "normal course" was identified. In the interview, the professor refers to such moments as "epistemological stops," as if a lecture were train travel and such moments thought of as stops made along the way to admire the view. These stops were often motivated by questions (Q) posed by the students and the amount of time and effort dedicated to each answer shows his desire to encourage students to ask them frequently. Moreover, the stops were frequently dedicated to philosophical discussions $(\mathrm{P})$ about the nature of physics and specific characteristics of physical knowledge. Although many people might think that philosophy of physics should belong to another course, the interviews reveal a different perspective: "The separation between physics and epistemology is artificial. I think that if you make an effort, you will realize that epistemology is everywhere."

Another special feature of the professor's discourse is the recursive use of explicit metacognitive remarks (Met). A conscious and careful monitoring of intellectual processes is an overall trademark of the lectures. This involves, among others, making the difficulties related to using mathematics in physics clear and explaining their reasons to the students. The professor justifies his attitude as follows: "There is something very intriguing in physics education. When people teach physics, they quite often forget how hard it was for them to understand something and teach it as if it were very easy and straightforward. [...] Perhaps they think that by doing so the students will understand better. I totally disagree and try to make the difficulties evident in my lectures."

The goal of this work was to analyze the lectures of a distinguished physics professor in order to characterize features of his didactical discourse to focus on the structural role of mathematics in physics. This case study reveals that teaching to use mathematics to reason about the physical world is a difficult and time-consuming task, which involves taking several aspects of the roles of mathematics into account. This stresses the complexity of concentrating on the structural dimension, as opposed to treating mathematics as a set of tools to calculate and describe (see technical versus structural dimensions in Table I).

Furthermore, this study provides an analytic tool that can be fruitful for conducting future comparative studies. The analysis of other lectures on the same topic may reveal considerable differences in the timelines generated by the software and imply different learning goals. The categorization system can also be used to analyze physics lessons in high school, textbooks, and other teaching materials. Moreover, the analytical tool can be implemented in teacher training programs to discuss the implications of different didactic approaches.

An envisaged future goal of this research is to establish objective criteria to characterize the notion of didactic quality of explanations, especially concerning the use of mathematics in physics. Considering the limitations of the study design and the early stage of the research in this area, it is only possible to formulate general hypotheses for 
further investigation. Additionally, it might be more prudent to speak about quality of explanations in situated contexts (e.g., explanation of a particular concept and solving of a particular problem). Nevertheless, an overall analysis of the lectures shows that the colorfulness of the profiles, i.e., the presence of numerous categories in a concept or problem-solving episode, may be an indicator of explanation quality. In a more traditional and technical approach, the discourse would likely be more rational and the timelines less colorful. The colorfulness identified in the lectures seems to be aligned with the complex and multifaceted character of the relationship between physics and mathematics underlined by several historical and epistemological studies [2-7].

Although it may be inferred that the multifaceted approach identified in this electromagnetism course is related to students' learning outcomes, the influence on learning is still subject to a deeper investigation. A possible research design would be to conduct thinking-out-loud problem-solving sessions with the students of this professor and investigate whether or not they frame problem solving similarly to the way it is done in the lectures. Another possibility would be to conduct similar analysis with students of other professors who teach in more traditional ways and compare their problem-solving process to those taught by the professor of this case study.

The overall message is to advocate in favor of changing the emphasis from a technical to a structural approach in physics instruction, which should help overcome the widespread notion of mathematics as a mere set of tools for problem solving in physics. The electromagnetism course investigated in this case study is full of examples of a professor who is committed to this task. Since the chief goal of the analyzed course is a deep understanding of the electromagnetic theory, whose elegant and powerful structure is mathematically expressed by Maxwell's equations, it seems fair to finish the paper with another recommendation of Maxwell to his students, in which he highlights the importance of "mastering a principle" (structural) in detriment of "getting up a formula" (technical):

I know the tendency of the human mind to do anything rather than think. None of us expect to succeed without labour, and I am sure we would all give a great deal of mental labour to get up our subjects. But mental labour is not thought, and those who have with great labour acquired the habit of application, often find it much easier to get up a formula than to master a principle. I shall endeavor to show you that principles are fertile in results, but the mere results are barren, and that the man who has thought out a principle may keep his mind clear of formulae, knowing that he could make any number of them when required. ([16], p. 671, my emphasis).

\section{ACKNOWLEDGMENTS}

This work is the result of research conducted at the University of São Paulo and the Technical University of Dresden. I thank Mauricio Pietrocola and Gesche Pospiech for providing the ideal research conditions, for their unconditional support, and for their constant availability for insightful discussions. Thanks also to Olaf Uhden, Debora Coimbra, Thaís Forato, Ana Maria Liblik, Jorge Nicolau, Pedro de Souza Neto, Fabiana Kneubil, Alex Belluco do Carmo, and Natalia Cury. I am grateful to the physics professor of this case study for authorizing the recording of his lectures. And I am indebted to Eric Kuo and Alex Maries for their critical comments. I also thank the three anonymous reviewers for their critical comments and most valuable suggestions. The financial support provided by CAPES (BEX 3481/09-3), FAPESP (2010/ 19276-8), and DAAD (4-month German course) is greatly acknowledged. Finally, I thank the Alexander von Humboldt Foundation for its financial support through BRA 1146348 STP.

\section{APPENDIX: TIMELINES AND BRIEF DESCRIPTION OF OTHER TEACHING EPISODES}

\section{Concept episode: Charge density $(1,2$, and 3 dimensions)}

\section{Lecture 5}

Total duration: 28 minutes.

Main characteristics: building (mathematizing) the concept of charge density; assumptions (idealizations, approximations) explicitly mentioned; several analogies with everyday life situations; intense use of gestures and pictorial representations; not a single moment of technical approach. Numerous metacognitive remarks and answers to students' questions. See Fig. 4.

\section{Concept episode: Meaning of Gauss's law (integral form)}

Lecture 14

Total Duration: 19 min.

Main characteristics: highlighting formal differences between open and closed surfaces; analogy charge-lamp; interpretation of Gauss's law: investigate the existence of charge in the interior of a volume by analyzing its surface. See Fig. 5.

\section{Concept episode: Ampère's law}

Lecture 31

Total Duration: $21 \mathrm{~min}$.

Main characteristics: careful presentation of the conventions involving Ampère's law (path orientation, mathematical surface supported by the path, direction of the normal); intensive use of visual representations; analogy 
with soap bubbles; interpretation of the Stokes theorem, and physical intuition of the curl. See Fig. 6

\section{Problem episode: Flux through a disk}

Lecture 12

Total duration: $35 \mathrm{~min}$.

Main characteristics: mathematization-technical-interpretation pattern; intense use of visual representations; careful and clear explanation of the relevant variables; long time dedicated to technical manipulations; emphasis on the deductive reasoning (Gauss's law as a "bigger truth" than Coulomb's law). See Fig. 7.

\section{Problem episode: Electric field of an infinite wire}

Lecture 15

Total duration: $10 \mathrm{~min}$.
Main characteristics: necessary conditions for applying Gauss's law; idealizations justified; formal differences between the infinite plane and wire; synthesis of the three cases (point, line, plane): relation between Gauss's law and spatial dimensions. See Fig. 8.

\section{Problem episode: Magnetic field created by a finite wire}

Lecture 19

Total duration: $20 \mathrm{~min}$.

Main characteristics: mathematization-technical-interpretation pattern; emphasis on representing relevant variables by vectors and on the cross product rules; formal differences between the radial symmetry of the electric field of a charge and the cylindrical symmetry of the magnetic field of a moving charge: velocity "breaks the symmetry" in one direction. See Fig. 9.

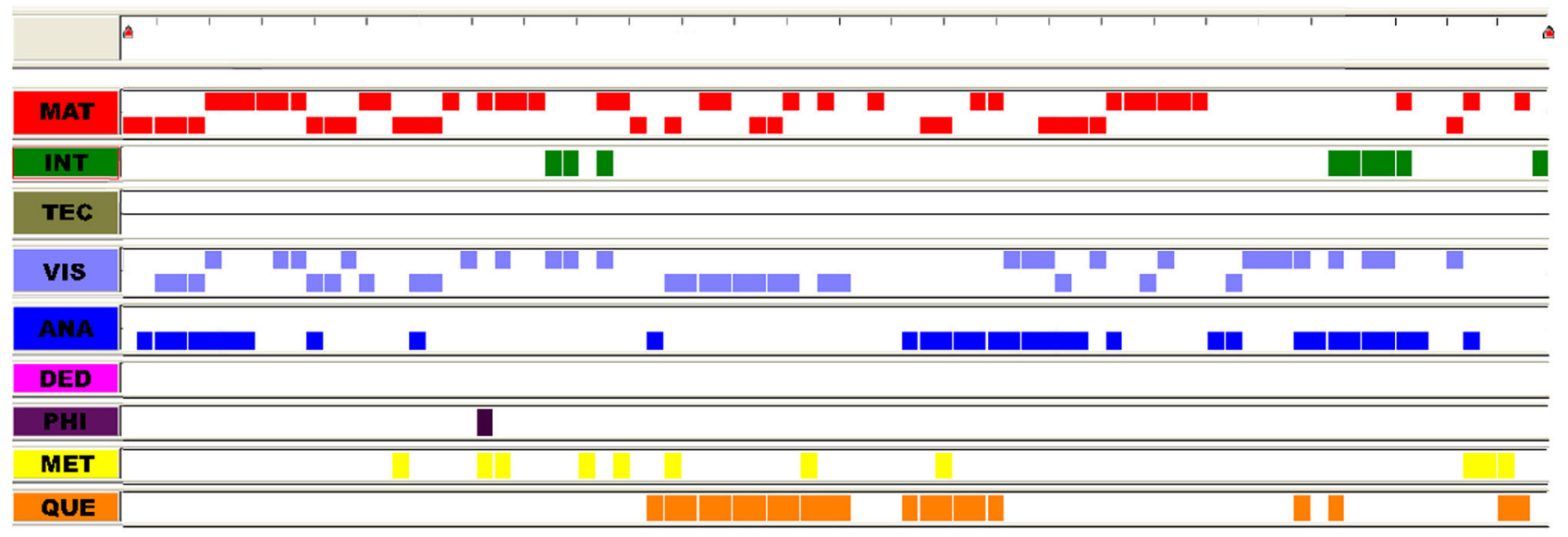

FIG. 4. Timeline of the charge density episode.

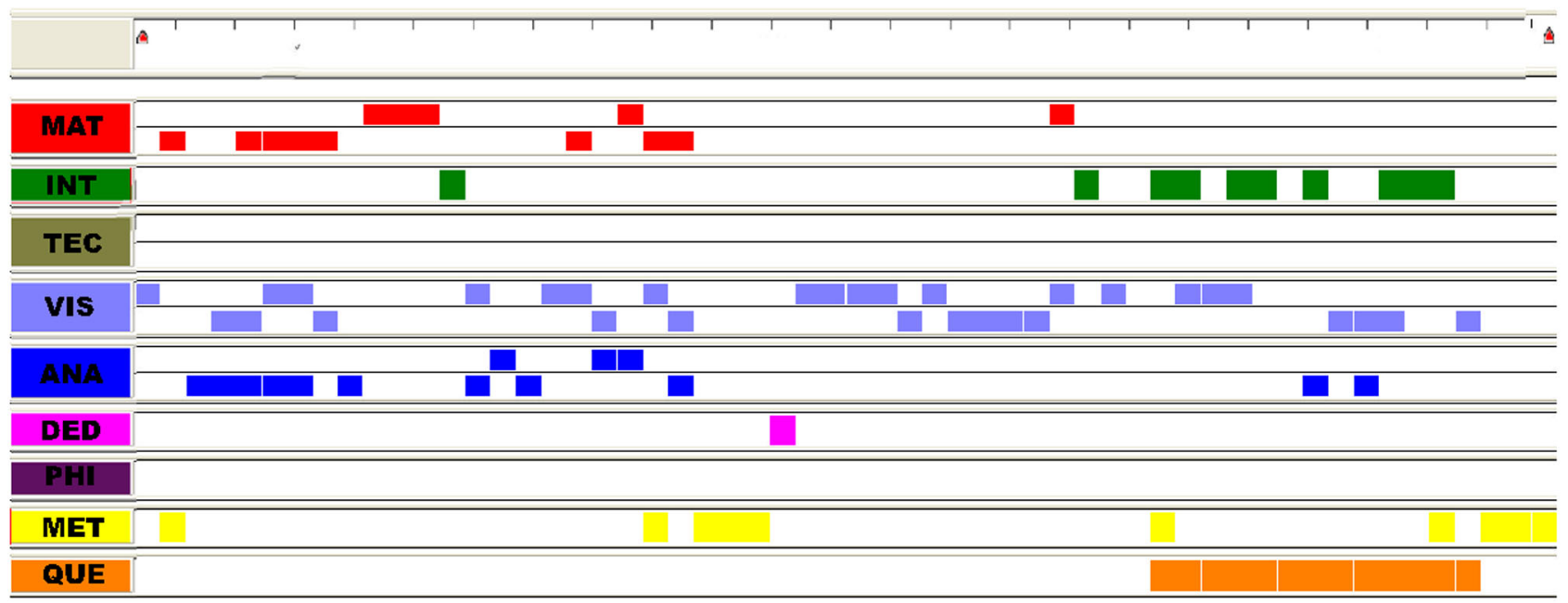

FIG. 5. Timeline of the Gauss's law episode. 


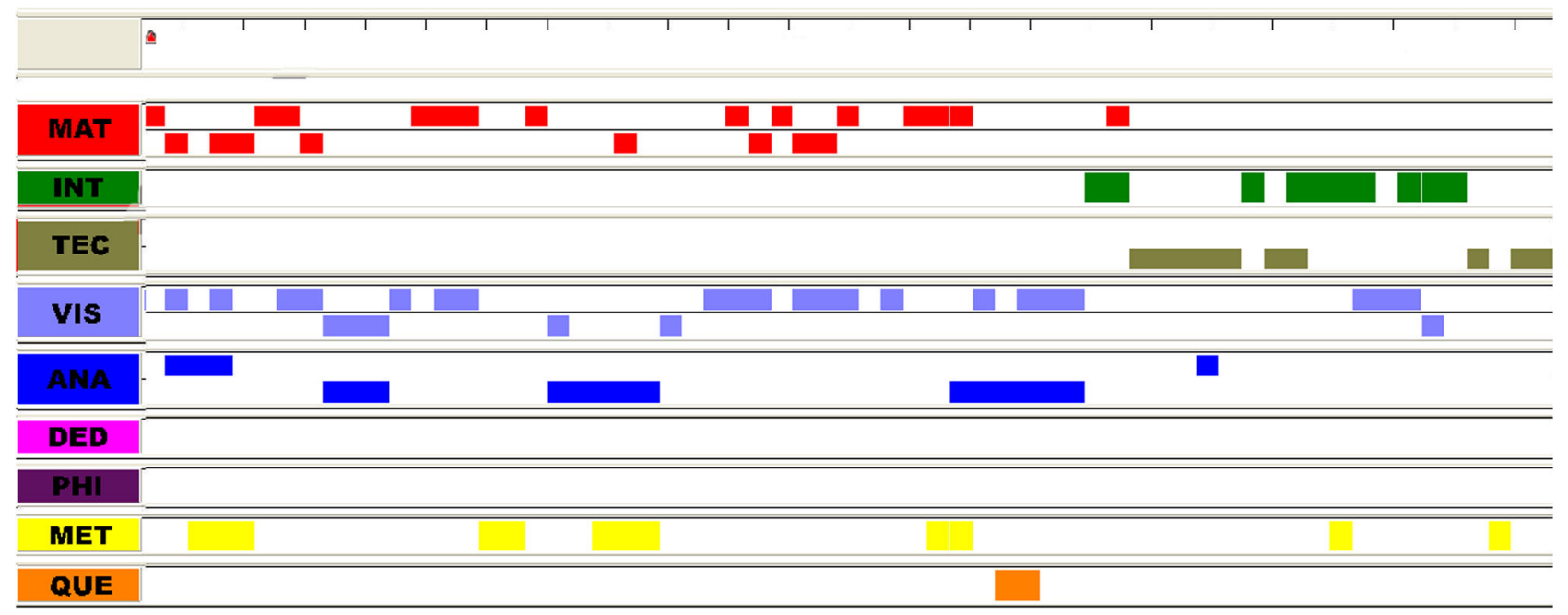

FIG. 6. Timeline of the Ampère's law episode.

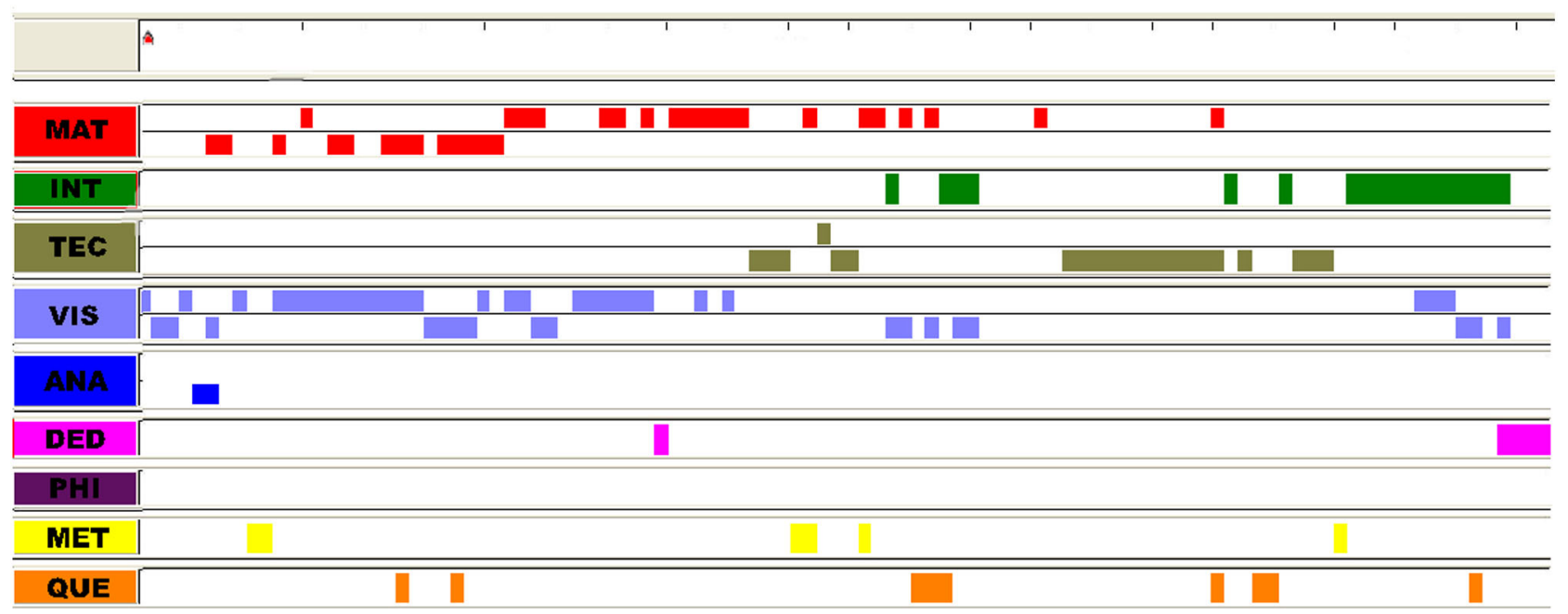

FIG. 7. Timeline of the flux through a disk problem episode.

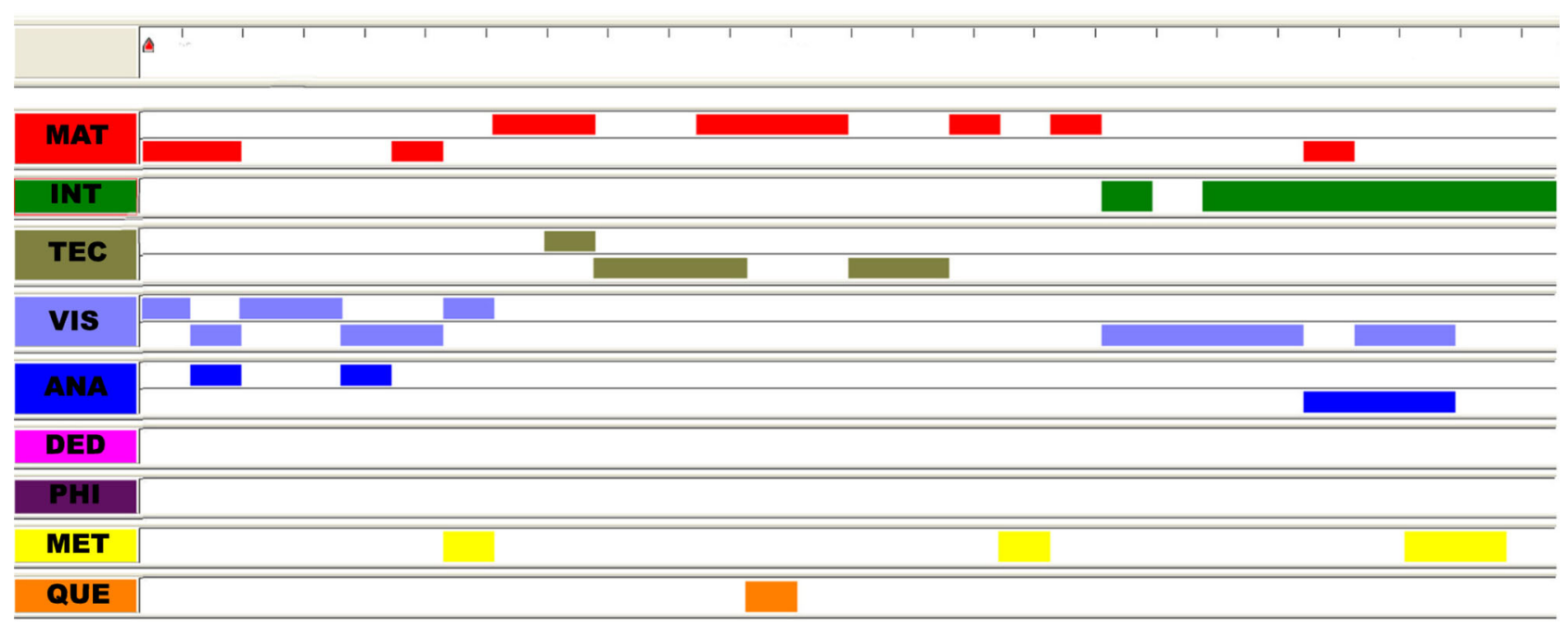

FIG. 8. Timeline of the electric field of an infinite wire problem episode. 




FIG. 9. Timeline of the magnetic field created by a finite wire problem episode.

[1] E. F. Redish and T. Bing, Using math in physics: Warrants and epistemological frames, in Proceedings of the 2009 GIREP-EPEC and PHEC International Conference, Leicester, England, edited by D. Raine, C. Hurkett, and L. Rogers, (University of Leicester, Leicester, UK, 2009) Vol. 2, pp. 71-76.

[2] M. Steiner, The Applicability of Mathematics as a Philosophical Problem (Harvard University Press, Cambridge, MA, 1998).

[3] S. Bochner, The Role of Mathematics in the Rise of Science (Princeton University Press, Princeton, NJ, 1981).

[4] Y. Gingras, What did mathematics do to physics?, Hist. Sci. 39, 383 (2001).

[5] R. P. Feynman, The Character of Physical Law (MIT Press, Cambridge, MA, 1985).

[6] M. Paty, in Constructivism and Practice: Towards a Social and Historical Epistemology, edited by C. Gould (Rowman and Littlefield, Lanham, MD, 2003), pp. 109-135.

[7] The Role of Mathematics in Physical Sciences, edited by G. Boniolo, P. Budinich, and M. Trobok (Springer, Dordrecht, 2005).

[8] B. Sherin, How students understand physics equations, Cognit. Instr. 19, 479 (2001).

[9] J. Tuminaro and E. F. Redish, Elements of a cognitive model of physics problem solving: Epistemic games, Phys. Rev. ST Phys. Educ. Res. 3, 020101 (2007).

[10] L. Walsh, R. Howard, and B. Bowe, Phenomenographic study of students problem solving approaches in physics, Phys. Rev. ST Phys. Educ. Res. 3, 020108 (2007).

[11] T. Bing and E. F. Redish, Analyzing problem solving using math in physics: Epistemological framing via warrants, Phys. Rev. ST Phys. Educ. Res. 5, 020108 (2009).

[12] E. Kuo, M. Hull, A. Gupta, and A. Elby, How students blend conceptual and formal mathematical reasoning in solving physics problems, Sci. Educ. 97, 32 (2013).
[13] N. S. Rebello, L. Cui, A. G. Benett, D. A. Zollman, and D. J. Ozimek, in Learning to Solve Complex Scientific Problems, edited by D. Jonassen (Lawrence Earlbaum Assoc., New York, 2007).

[14] E. Bagno, H. Berger, and B.S. Eylon, Meeting the challenge of students' understanding of formulae in high-school physics: A learning tool, Phys. Educ. 43, 75 (2008).

[15] C. Angell, P. M. Kind, E. K. Henriksen, and Ø. Guttersrud, An empirical-mathematical modeling approach to upper secondary physics, Phys. Educ. 43, 256 (2008).

[16] The Scientific Letters and Papers of James Clerk Maxwell, edited by P. Harman (Cambridge University Press, Cambridge, England, 1990), Vol. 1, pp. 1846-1862.

[17] M. Pietrocola, in Connecting Research in Physics Education with Teacher Education, edited by M. Vicentini and E. Sassi (University of Amsterdam, Amsterdam, 2008), Vol. 2, p. 452.

[18] G. Pospiech, Promoting the competence of mathematical modeling in physics lessons, in Proceedings of the 2006 GIREP Conference: Modelling in Physics and Physics Education, Amsterdam, edited by E. van den Berg, A. Ellermeijer, and O. Slooten (2006), pp. 587-595.

[19] O. Uhden, R. Karam, M. Pietrocola, and G. Pospiech, Modelling mathematical reasoning in physics education, Sci. \& Ed. 21, 485 (2012).

[20] H. Hudson and W. McIntire, Correlation between mathematical skills and success in physics, Am. J. Phys. 45, 470 (1977).

[21] L. Hoffmann, P. Häußler, and M. Lehrke, Die IPNInteressenstudie Physik Kiel: IPN (1998).

[22] D. Hestenes, Oersted Medal Lecture 2002: Reforming the mathematical language of physics, Am. J. Phys. 71, 104 (2003). 
[23] S. Chasteen, S. Pollock, R. Pepper, and K. Perkins, Thinking like a physicist: A multi-semester case study of junior-level electricity and magnetism, Am. J. Phys. 80, 923 (2012).

[24] E. F. Redish, arXiv:physics/0608268.

[25] It is important, however, not to associate the technical dimension with mathematics as a discipline or with something that has to be learned in mathematics classes. To promote meaningful understanding and avoid instrumental memorization of rules is a major goal that has been systematically addressed by mathematics educators. In fact, several similar dichotomies or dualities can also be found in the mathematics education literature. Some examples are instrumental and procedural understanding [26], conceptual and procedural [27], abstract and algorithmic [28], operational and structural [29], and dialectic and algorithmic [30].

[26] R. Skemp, Relational understanding and instrumental understanding, Math. Teach. 77, 20 (1976).

[27] Conceptual and Procedural Knowledge: The Case of Mathematics, edited by J. Hiebert (Lawrence Erlbaum Associates, Hillsdale, NJ, 1986).

[28] P. Halmos, Pure thought is better yet..., College Math. J. 16, 14 (1985).

[29] A. Sfard, On the dual nature of mathematical conceptions: Reflections on processes and objects as different sides of the same coin, Educ. Stud. Math. 22, 1 (1991).

[30] P. Henrici, The influence of computing on mathematical research and education, in Proceedings of the Symposia in Applied Mathematics, edited by J. Lasalle (American Mathematical Society, Providence, RI, 1974), Vol. 20.

[31] T. Ellermeijer and A. Heck, Differences between the use of mathematical entities in mathematics and physics and the consequences for an integrated learning environment, in Proceedings of the First International GIREP Seminar: Developing Formal Thinking in Physics, 2001, edited by M. Michelini and M. Cobal (Forum, Udine, 2001), pp. 52-72.

[32] "Unlike the experimenter who manipulates variables to determine their causal significance or the surveyor who asks standardized questions of large, representative samples of individuals, the case study researcher typically observes the characteristics of an individual unit-a child, a clique, a class, a school or a community. The purpose of such observation is to probe deeply and to analyze intensively the multifarious phenomena that constitute the life cycle of the unit with a view to establishing generalizations about the wider population to which that unit belongs" (Ref. [33], p. 258).

[33] J. Gerring, Case Study Research: Principles and Practices (Cambridge University Press, New York, 2007).

[34] L. Cohen and L. Manion, Research Methods in Education (Routledge, London, 1989).

[35] Students who take this course have already taken Calculus 3 and are familiar with the vector calculus tools. This enables the professor to present Maxwell's equations using both the integral and the differential formalism.

[36] I had access to documents that show how many times the professor gave this course since 1975 and found out that we videotaped the 16th time he taught the introductory course on electromagnetism in the department. This reinforces his comprehensive teaching experience and attests to the pertinence of the case study.

[37] P. A. Tipler, Physics (Worth, New York, 1982), 2nd ed.

[38] D. Halliday, R. Resnick, and J. Walker, Fundamentals of Physics (Wiley, London, 2011).

[39] D. Giancoli, Physics for Scientists and Engineers (Prentice-Hall, Upper Saddle River, NJ, 2000), Vol. 2.

[40] R. A. Serway and J. W. Jewett, Physics for Scientists and Engineers with Modern Physics (Thomson, Belmont, CA, 2008), 7th ed.

[41] The maximum score on the exams is 10 . The average score $M$ must be greater than or equal to 5.0 for approval.

[42] R. Karam, Using mathematics as a reasoning instrument in physics instruction: A theoretical tool for the analysis of didactic approaches, Ph.D. thesis (text in Portuguese), University of São Paulo, 2012 (http://www.teses.usp .br/teses/disponiveis/48/48134/tde-29052012-134910/en .php).

[43] Information about the software can be obtained at http:// www.dervideograph.de/enhtmStart.html.

[44] The Power of Video Studies in Investigating Teaching and Learning in the Classroom, edited by T. Janik and T. Seidel (Waxmann-Verlag, Göttingen, 2009).

[45] M. Kline, Mathematics and the Physical World (Thomas Y. Crowell Co., New York, 1959).

[46] [...] if one seeks to study motion on and near the earth, say, and observes the fall of many different sizes, shapes, and weights of bodies, or the flight of a variety of projectiles, he will end up with a mass of observations from which no clear pattern or principle will emerge. Galileo did otherwise. He attempted, insofar as thought and experiment would permit, to strip away all the incidental and minor effects. To eliminate the effects of friction he considered smooth balls rolling down a smooth slope. Having discovered that pendulum motion is little affected by air resistance, he studied pendulum motion to obtain fundamental principles of motion. This approach is precisely the one the mathematician makes when, for example, he studies geometrical figures. He strips away molecular structure, color, and thickness to get at the basic properties of these figures. Thus, insofar as he observed and experimented, Galileo idealized just as the mathematician does and thereby penetrated the phenomena to obtain the basic physical principles (Ref. [45], p. 176, my emphasis).

[47] The lectures were given in Portuguese. Therefore, all the transcripts presented in this paper are translations into English made by the author.

[48] J. Lemke, Reading Science, edited by J. Martin and R. Veel (Routledge, London, 1998), pp. 87-113.

[49] This categorization is also inspired by Hesse's distinction between formal and material analogies [50].

[50] M. Hesse, Models in physics, Br. J. Philos. Sci. IV, 198 (1953).

[51] R. P. Feynman, R. B. Leighton, and M. Sands, The Feynman Lectures on Physics (Addison-Wesley, Reading, MA, 1964), Vol. 2. 
[52] A. Rivadulla, The Role of Mathematics in Physical Sciences, edited by G. Boniolo, P. Budinich, and M. Trobok (Springer, Dordrecht, 2005), pp. 161-178.

[53] A. Einstein, Mein Weltbild (Ullstein Tachenbücher-Verlag, Frankfurt, 1956).

[54] This metaphor was suggested by the professor in one of the interviews.
[55] M. V. J. Veenman, B. H. A. Van Hout-Wolters, and P. Afflerbach, Metacognition and learning: Conceptual and methodological considerations, Metacogn. Learn. 1, 3 (2006).

[56] C. Singh, Student understanding of symmetry and Gauss's law of electricity, Am. J. Phys. 74, 923 (2006). 